\title{
Measurement of the $\tau^{-} \rightarrow \pi^{-} \nu_{\tau} e^{+} e^{-}$branching fraction at Belle
}

\section{Yifan Jin*}

The University of Tokyo, 7-3-1 Hongo Bunkyo-ku, Tokyo 113-0033, Japan

E-mail: jin@hep.phys.s.u-tokyo.ac.jp

\section{Denis Epifanov}

Budker Institute of Nuclear Physics, akademika Lavrentieva prospect, Novosibirsk, 630090,

Russian Federation

\section{Hiroaki Aihara}

The University of Tokyo, 7-3-1 Hongo Bunkyo-ku, Tokyo 113-0033, Japan

\section{On Behalf of the Belle Collaboration}

Preliminary result of the measurement of the branching fraction of rare tau decay, $\tau^{-} \rightarrow$ $\pi^{-} v_{\tau} e^{+} e^{-}$, is presented. A $562 \mathrm{fb}^{-1}$ data set collected with Belle detector at the KEKB asymmetric-energy $\mathrm{e}^{+} \mathrm{e}^{-}$collider is used. The branching fraction of $\tau^{-} \rightarrow \pi^{-} e^{+} e^{-} v_{\tau}$ is measured to be $\mathscr{B}\left(\tau^{-} \rightarrow \pi^{-} e^{+} e^{-} v_{\tau}\right)=(2.11 \pm 0.19 \pm 0.30) \times 10^{-5}$, where the first uncertainty is statistical and the second is systematic. The measured result is consistent with the theoretical prediction from the Standard Model and could facilitate searches for physics beyond the Standard Model.

The 39th International Conference on High Energy Physics (ICHEP2018)

4-11 July, 2018

Seoul, Korea

${ }^{*}$ Speaker. 


\section{Introduction}

The Belle detector, which was installed at the interaction point of the electron-positron asymmetric energy collider (KEKB), was working not only as a B-factory but also as a Tau-factory. The world's largest statistics of tau leptons was collected at Belle, which makes the measurement of branching fraction for rare decays $\left(\mathscr{B} \sim 10^{-5}\right)$ of tau possible. We herein present the preliminary result of the measurement of the branching fraction of rare decay $\tau^{-} \rightarrow \pi^{-} v_{\tau} e^{+} e^{-}$using a $562 \mathrm{fb}^{-1}$ data set collected at Belle. The measured result could be used to validate the calculation [1] from Resonance Chiral Theory, a prevailing phenomenological approach to describe strong interaction at the energy region below tau mass, and to facilitate searches for physics beyond the Standard Model, e.g., sterile neutrinos and lepton flavour violating decays.

\section{Analysis strategy and preliminary results}

The analysis is carried out as a blind analysis. MC samples for processes that can occur at $\Upsilon(4 S)$ are exploited. Embedding the signal mode in TAUOLA, a signal generator is prepared according to [1]. A standard Belle $\tau \tau$ preselection is employed first, followed by further selection of the events in which one $\tau$ decays into the mode of concern while the other $\tau$ decays into the oneprong. After optimisation of the selections, the main background comes from the $\tau^{-} \rightarrow \pi^{-} \pi^{0} v_{\tau}$ decay which is generated based on the Belle pion form factor [2]. A sideband (signal box) is defined by the invariant mass of 3 prongs $\left(\pi^{-}, e^{+}, e^{-}\right)$ranging between $[0,1] \mathrm{GeV}([1.05,1.8] \mathrm{GeV})$, to validate MC simulation.

After opening the signal box, 676 events are observed in the signal box of $\tau^{-} \rightarrow \pi^{-} v_{\tau} e^{+} e^{-}$ with a background expectation of $478 \pm 23$ events and 689 events are observed in the signal box of the charge-conjugate mode, $\tau^{+} \rightarrow \pi^{+} \bar{v}_{\tau} e^{+} e^{-}$, with a background expectation of $476 \pm 22$ events, which gives rise to a branching fraction $\mathscr{B}\left(\tau^{-} \rightarrow \pi^{-} e^{+} e^{-} v_{\tau}\right)=(2.11 \pm 0.19 \pm 0.30) \times 10^{-5}$, where the first uncertainty is statistical and the second one is systematic. The systematic uncertainty is estimated as $14.4 \%$ stemming from MC size (3.74\%), $\tau \tau$ cross section $(0.3 \%)$, trigger $(1.16 \%)$, $\pi^{0}$ veto (1.86\%), accuracy of branching fractions of BKG (4.42\%), luminosity (4.66\%), tracking $(4.66 \%)$ and particle identification $(11.14 \%)$.

\section{Conclusion}

Using a $562 \mathrm{fb}^{-1}$ data set collected with Belle detector at the KEKB asymmetric-energy $\mathrm{e}^{+} \mathrm{e}^{-}$collider, the branching fraction of the $\tau^{-} \rightarrow \pi^{-} e^{+} e^{-} v_{\tau}$ decay is measured for the first time, $\mathscr{B}\left(\tau^{-} \rightarrow \pi^{-} e^{+} e^{-} v_{\tau}\right)=(2.11 \pm 0.19 \pm 0.30) \times 10^{-5}$.

\section{References}

[1] P. Roig, et al., Weak radiative pion vertex in $\tau^{-} \rightarrow \pi^{-} v_{\tau} l^{+} l^{-}$decays, Physical Review D, 2013, 88(3): 033007.

[2] M. Fujikawa, H. Hayashii, S. Eidelman, et al., High-statistic study of the $\tau^{-} \rightarrow \pi^{-} \pi^{0} v_{\tau}$ decay, Physical Review D, 78 (2008) 072006. 